\title{
Thermal effects of different kind influencing lubricated non-conformal contacts
}

\author{
E. Ciulli \\ Dipartimento di Ingegneria Meccanica, Nucleare e della Produzione \\ University of Pisa \\ Largo Lucio Lazzarino, 56126 Pisa, Italy \\ Tel.: +39 050 2218061; fax: +39 050 2218065; e-mail: ciulli@ing.unipi.it
}

\begin{abstract}
Three steel spherical specimens with different diameters are tested against a glass disc under the same working conditions in several rolling speeds and slide-to-roll ratios. The same tests are carried out with the specimens connected to a steel shaft with and without a ceramic insert purposely designed for limiting the transfer of the heat generated by the shaft seals and bearings to the specimens, in order to evaluate the influence of the experimental apparatus on friction results. A general increase in friction is found when the ceramic insert is used probably related to a local greater lubricant viscosity inside the contact due to the reduction in the specimen surface temperature. However, the configuration of the experimental apparatus does not influence in a significant way the shape of the friction curves as a function the entraining speed and of the slide-to-roll ratio. The analysis of the interference images of the contact shows that the thermal insulation due to the ceramic insert does not affect particularly the film thickness.
\end{abstract}

Keywords: elastohydrodynamic lubrication, friction, film thickness, thermal effects

\section{Introduction}

The determination of the friction coefficient $f$ of a lubricated contact is not an easy task. As well known, the lubrication regime (boundary, mixed and full fluid film) influences the trend of the friction coefficient. Friction is related to important quantities for a lubricated contact, such as viscosity, speed and load. Their effect is well represented by the classical Stribeck curve. However, other quantities can affect $\mathrm{f}$, for instance materials and surface roughness, particularly for boundary and mixed regimes, and the rheological behaviour of the lubricant.

For typical non-conformal lubricated contact, as the ones occurring between gear teeth and cam and follower, mainly working under classical elastohydrodynamic lubrication conditions (EHL), additional quantities have a significant influence. One of these is the slide-to-roll ratio $\mathrm{S}$, the ratio of the difference and the mean value of the surface velocities of the two bodies in contact. Being a measure of sliding in relation to rolling, its influence is also related to thermal effects.

The influence of $\mathbf{S}$ on the film thickness is relatively well understood, while its influence on friction is more complex to understand. For instance, in [1] and [2] formulas are developed where the deviation from the pure rolling film thickness values can be calculated by a correction factor and explained by inlet heating. Experimental measurements of the film thickness for different slide-to-roll ratios $S$ are reported in [3], [4], [5] and [6]. The inlet heating and rise in bulk temperature could explain the change in film thickness by different $S$ only to some extent. The formation of a dimple in the central part of the contact is shown under high sliding conditions if materials with different thermal properties are used. Agreement between experimental findings and numerical simulations can be found by solving also the energy equation and explaining the occurrence of dimples with the so-called temperature viscosity wedge effect [7]. The temperature-viscosity wedge action is caused by the temperature variation across the oil film. In addition, there is an increase in oil temperature at the entrance of the contact due to the heat produced by the compression work and the shearing of the oil. The degree of both effects depends on the thermal properties of the contacting materials and the lubricant.

The above mentioned aspects can give some explanations for understanding the friction trends that can be found when varying in particular the speed, but sometimes these are not sufficient. An elastohydrodynamic contact has usually very small dimensions, but what it is occurring inside it is very 
complex and several factors can influence the friction trends. Interesting insights on friction are furnished in [8] by detailed mapping of the local temperatures of the lubricant and of the bodies surfaces. By combining infrared camera measurements and moving heat theory, the trend of the local friction coefficient inside the contact can be evaluated. A general good agreement was found between the traction values evaluated from integration of the local shear stress values and the ones directly measured with conventional methods. Another complex aspect important for friction is the heat partition between the bodies. As shown in [9], the heat partition depends on several aspects, as the relative speeds of the contacting bodies and their thermal diffusivity. The influence of the surface temperature of the solids on friction and film thickness is investigated from a theoretical point of view in [10]. Results are greatly influenced by the lubricant model used, particularly if Newtonian or non-Newtonian. The thermal problem in EHL contacts is also solved in a simplified manner in [11] for the calibration of the rheological parameters of several lubricants by using experimental results.

The friction behaviour of the ball a disc lubricated contact has been investigated in many different conditions in [12], [13] and [14]. Some intersections of the friction trends for different slide-to-roll ratios $\mathrm{S}$ in the Stribeck-like diagrams, where the friction coefficient $\mathrm{f}$ is plotted as a function of the entraining velocity $\mathrm{u}$, were found and possible explanations related to thermal effects were suggested. The intersections of the friction curves were found for $S<0$ (disc running faster than the specimen) but also for $\mathrm{S}>0$ (ball running faster). It was found that there is also a difference in the friction coefficient trends for negative and positive slide-to-roll ratios, whenever the contacting materials have different thermal properties. However, it was not possible to distinguish between thermal effects caused by the actual tribological phenomenon (such as the temperature viscosity wedge action) and thermal effects caused by the characteristics of the test rig, as the heating of the specimen due to the shaft bearings and seals friction losses. In order to separate these effects, a shaft with a thermal insulation for diminishing the heating effects particularly at high rpm has been developed and tested. A preliminary analysis of some friction results has been reported in [15]. Friction values are always greater when the shaft with the ceramic insert is used rather than in the case with the all steel shaft.

This work is focused on the friction differences due to the different heating of the specimens tested in the same working conditions of load and speed with the two shafts, with and without the ceramic insert. The friction results obtained are analysed in a deeper manner than in [15] and are also put in relation to the film thickness.

\section{Experimental procedure}

The experimental work has been carried out using an apparatus able to measure the friction force and the film thickness in a lubricated contact. The tests have been performed using spherical specimens and a glass disc. The same load, lubricant and temperature have been used for all tests carried out under several speed conditions as described in the following.

\subsection{Details of the experimental set-up}

The used experimental rig is shown in Fig.1.

The lubricated contact is formed between the plane surface of the disc and the specimen. Both the disc and the specimen are driven by separate electric motors connected to the output of a digital acquisition board. The load is applied by dead weights thanks to a lever system with a gas bearing in the fulcrum, which makes it possible the measurement of the friction force with a load cell that restrains the movement of the whole specimen group along the bearing axis. Optical interferometry is used for film thickness and shape detection using a microscope connected to a computer-controlled camera. The inlet and outlet temperatures of the lubricant, the velocity of disc and specimen, and the traction force acting on the specimen are recorded by a data acquisition system. The test rig is controlled with programs purposely developed in the LabView ${ }^{\circledR}$ environment. 

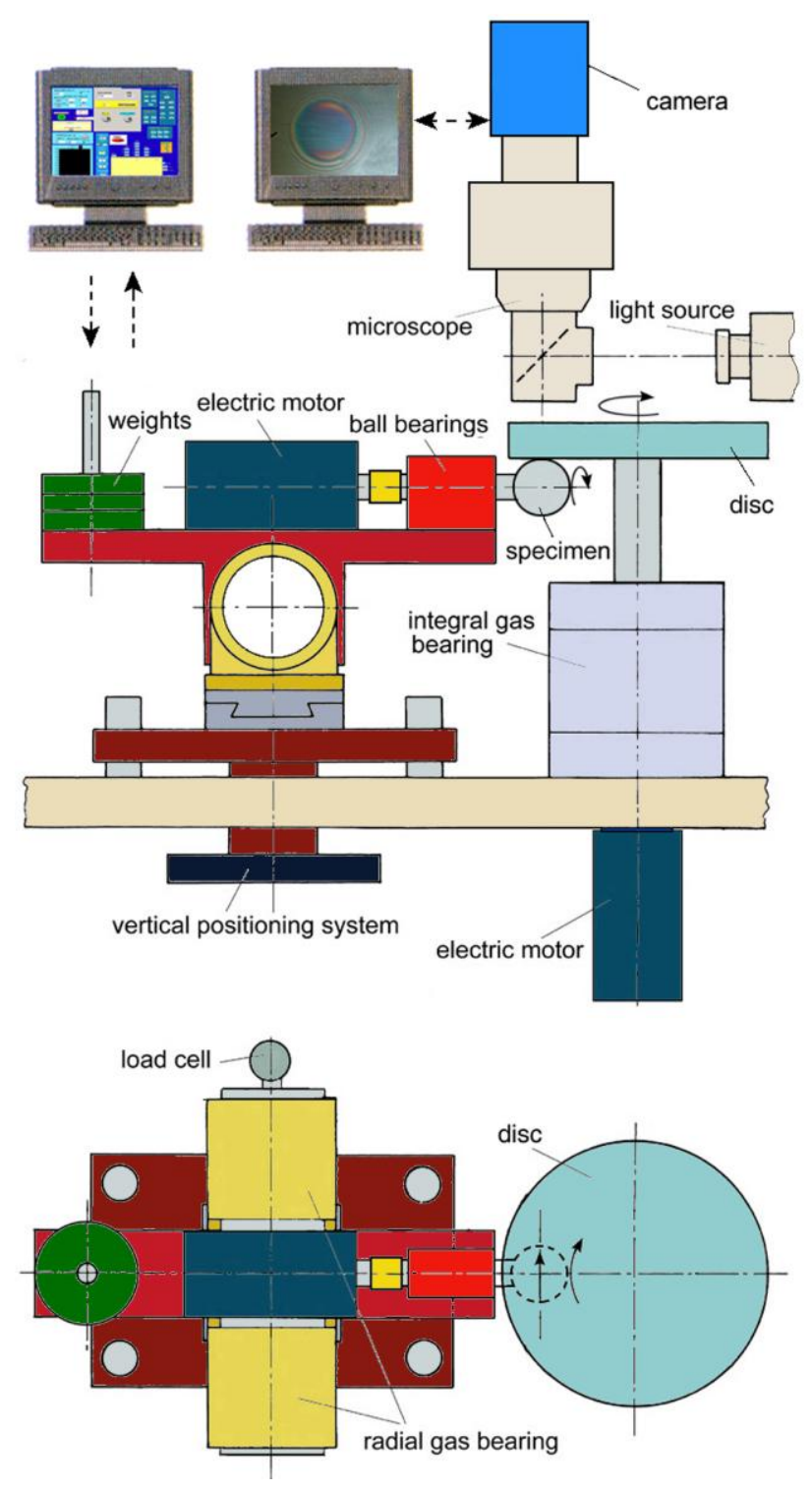

Fig. 1. The experimental apparatus.

The specimen steel shaft is supported by a couple of preloaded angular contact ball bearings. The bearing cavity is delimited by two radial shaft seals. Both the bearings and the seals produce a heating of the shaft that can increase the temperature of the specimen. In order to reduce the influence of this heating on the lubricated contact, a shaft with a ceramic insert has been designed and realized. The usual steel shaft and the one with the ceramic insert are shown in Fig.2.

\subsection{Test conditions}

Three spherical specimens of 100Cr6 (AISI 52100) with different diameters have been tested against a glass disc with a root mean square roughness $\mathrm{R}_{\mathrm{q}}=0.01 \mu \mathrm{m}$. The specimens, labelled with S1, S3 and S6, are shown in Fig.3.

Specimens and disc have been used in various tests, connected both to the usual steel shaft and to the one with the ceramic insert. The three specimens have been tested under the same conditions of load, peripheral speed and oil temperature, in order to verify the influence of the diverse rotational speeds in connection to the heating development.

The entraining velocity $\mathrm{u}=\left(\mathrm{u}_{\mathrm{s}}+\mathrm{u}_{\mathrm{d}}\right) / 2$, with $\mathrm{u}_{\mathrm{s}}$ and $\mathrm{u}_{\mathrm{d}}$ surface velocity of the specimen and of the disc respectively, has been varied from 0.05 to $0.5 \mathrm{~m} / \mathrm{s}$. The slide-to-roll ratio $S=2\left(u_{s}-u_{d}\right) /\left(u_{s}+u_{d}\right)$ has been varied from -1.8 to +1.8 . 

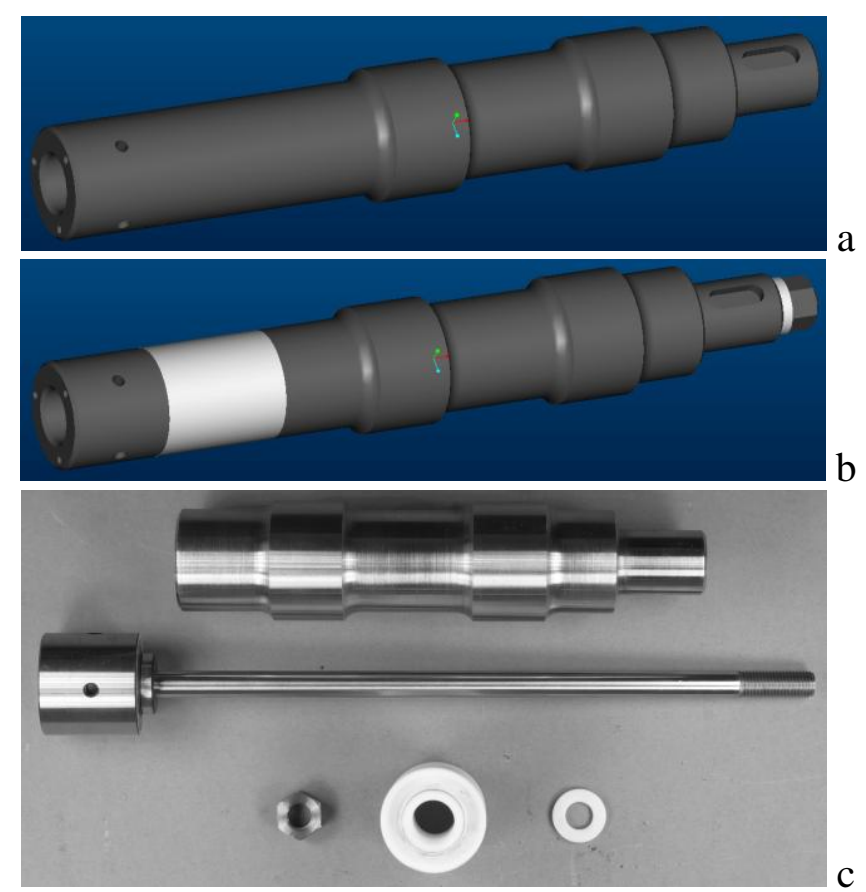

Fig. 2. 3D view of the specimen steel shaft (a) and of the shaft with a ceramic insert (b); picture of the components of the shaft with ceramic insert (c).

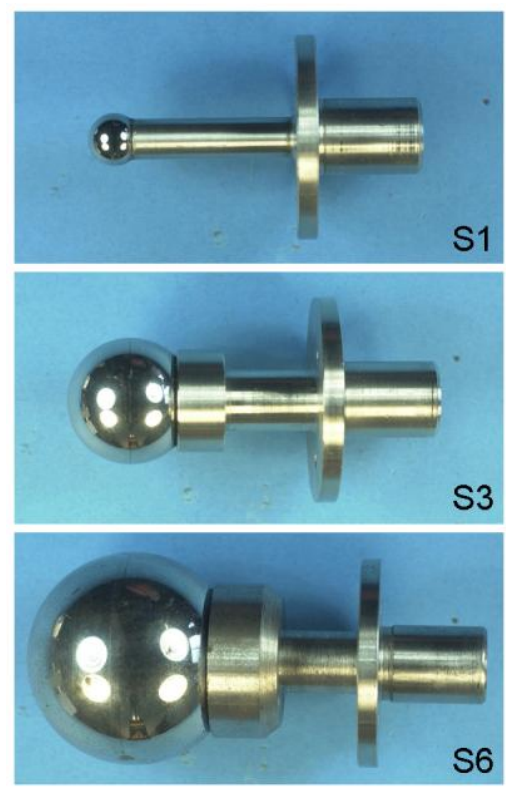

Fig. 3. Pictures of the specimens tested.

A neopentyl polyol ester base lubricant with a dynamic viscosity $\eta_{0}=0.057$ Pas at the test temperature $\left(20^{\circ} \mathrm{C}\right)$ has been employed. The load has been kept constant at $26.8 \mathrm{~N}$, that corresponds to the Hertzian pressures and radii reported in Tab.1, where also some details of the specimens are reported.

\begin{tabular}{lccc}
\hline Specimen & $\mathrm{S} 1$ & $\mathrm{~S} 3$ & $\mathrm{~S} 6$ \\
\hline Diameter $[\mathrm{mm}]$ & 10.319 & 24.606 & 60.321 \\
Roughness $\mathrm{R}_{\mathrm{q}}[\mu \mathrm{m}]$ & 0.010 & 0.016 & 0.016 \\
Hertzian pressure $[\mathrm{GPa}]$ & 0.893 & 0.500 & 0.275 \\
Hertzian radius $[\mathrm{mm}]$ & 0.120 & 0.160 & 0.216 \\
\hline
\end{tabular}

Table 1. Specimens and contact data. 


\section{Experimental results}

Several values of the entraining velocity $u$ and of the slide-to-roll ratio $S$ have been tested: $u=0.05,0.10$, $0.15,0.20,0.25,0.30,0.40,0.50 \mathrm{~m} / \mathrm{s}, \mathrm{S}=0, \pm 0.25, \pm 0.5, \pm 0.75, \pm 1, \pm 1.25, \pm 1.5, \pm 1.8$.

Due to the big amount of data recorded, only the results obtained with some selected values of $\mathrm{u}$ and $\mathrm{S}$ have been reported in this work: three values of the entraining velocity $(\mathrm{u}=0.1,0.2$ and $0.4 \mathrm{~m} / \mathrm{s})$ and six values of the slide-to-roll ratio $(S=-1.8,-0.75,-0.25,+0.25,+0.75,+1.8)$. Note that positive values of $S$ mean that the specimen is running faster than the disc while negative values that the disc is running faster than the specimen. Under pure rolling conditions $(S=0)$ friction values are negligible and they have been considered as reference values corresponding to zero friction for the purpose of this work.

\subsection{Friction}

A full picture of the results obtained with the different specimens is given by the diagrams shown in Fig.4. In these diagrams the friction coefficient is plotted as a function of the entraining velocity $u$ in the classical Stribeck-like diagrams. Each diagram refers to the results recorded for a single specimen connected with the steel shaft (diagrams on the left) and with the shaft with the ceramic insert (diagrams on the right).

Values of $\mathrm{f}$ generally increase by increasing the absolute value of $\mathrm{S}$ for both shafts. However, some opposite effects, corresponding to intersections of the friction curves for different $S$, can be noted for the highest values of $u$ for $S<0$ as found in [13] and [14]. This effect that decreases by increasing the specimen dimensions can be related to thermal effects that are bigger for the smallest specimen (S1) and negligible for the biggest one (S6). An explanation can be given by the different film thickness and Hertzian pressure for the three specimens. The smallest specimen undergoes the highest Hertzian pressure and the smallest film thickness, which is related to higher friction, as confirmed by the values of the friction coefficient (note that different scales are used for the vertical axes of the diagrams of Fig.4 for each specimen). Higher pressure and friction produce higher losses and therefore higher heating. The results obtained using the shaft with the ceramic insert generally show higher friction values than the ones obtained when the steel shaft is used. This can be related to a lower temperature of the specimen and probably of the lubricant, producing a higher viscosity. Again, the effect is bigger for the smallest specimen $\mathrm{S} 1$ that needs a higher rotational speed than the other ones to reach a certain peripheral velocity. This produces a higher heating of the specimen and therefore the insulation effect of the ceramic insert is more effective, particularly for $\mathrm{S}>0$, when the specimen is rotating faster. This effect is not always evident for the biggest specimen S6, but it is worth mentioning that the very low values of $\mathrm{f}$ recorded for this specimen are close to the precision of the measurement; in addition the rotational speed of the specimen is close to the motor minimum rotational speed. Therefore errors on friction force and speed are in proportion higher in this case.

By comparison of the curves for positive and negative values of $\mathrm{S}$, it can be noted a greater value of $\mathrm{f}$ for $S>0$ than for $S<0$, especially when thermal effects due to heat conduction through the shaft are smaller as in the case of the ceramic insert. This is consistent with the lower film thickness for positive values of $S$ than for negative ones reported for instance in [3] and [13], that can be put in relation to the lower thermal conductivity of the glass disc in comparison with the one of the steel balls.

It is worth mentioning that the present test conditions are significantly different from the ones reported in [12], [13] and [14] regarding with the viscosity of the lubricant, that is much lower in this case. However, the intersections of the friction curves are still present for the specimen $\mathrm{S} 1$ when $\mathrm{S}<0$ both for the steel shaft and the shaft with the ceramic insert. 

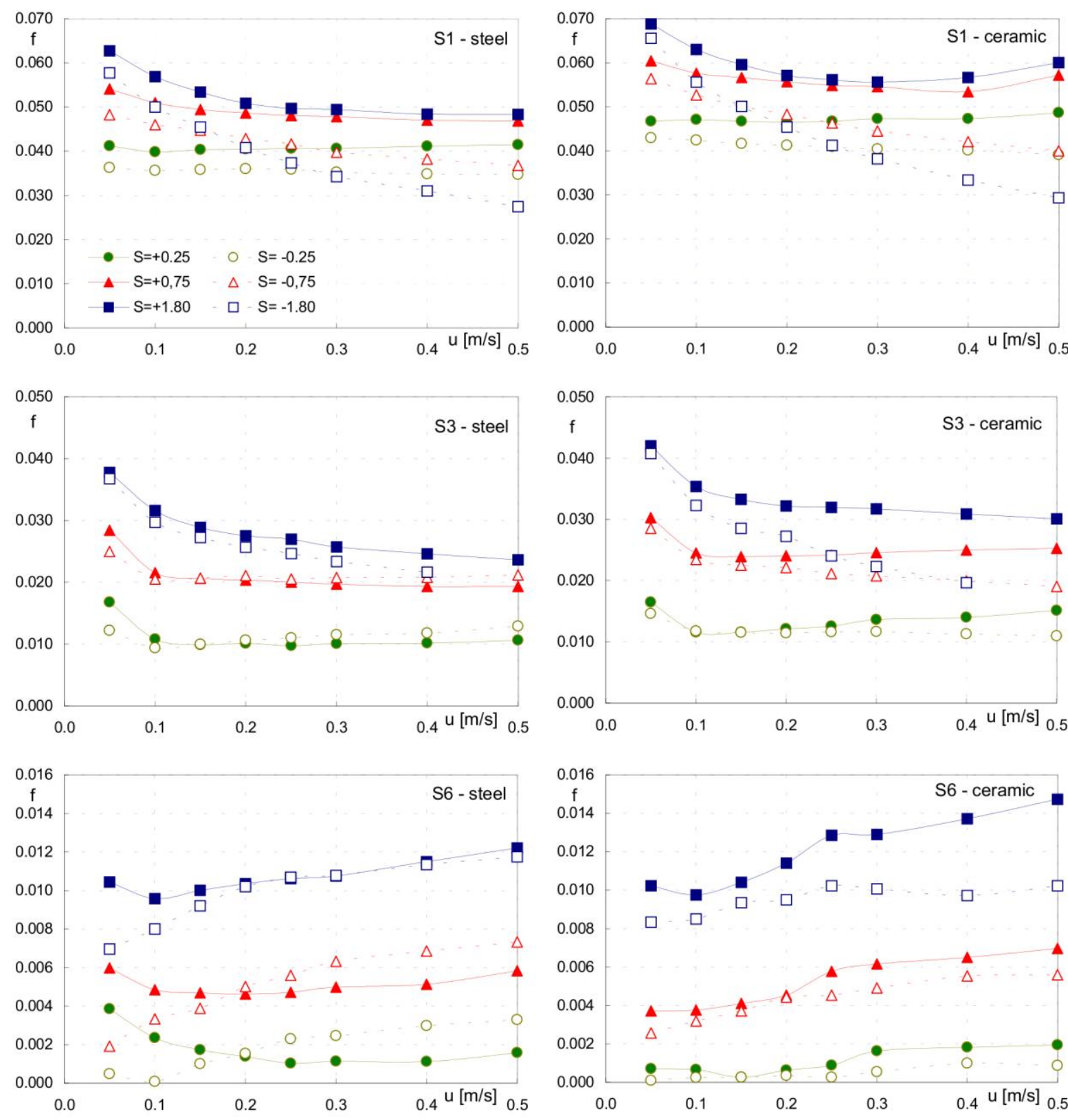

Fig. 4. Measured friction trends as a function of the entraining velocity $u$ for the three specimens tested for three positive and negative values of the slide-to-roll ratio S. Steel shaft (diagrams on the left) and shaft with the ceramic insert (diagrams on the right).

\subsection{Film thickness}

The main aim of this work was the investigation of thermal effects on friction. However, interference images have been recorded for every tested condition using the high-speed camera. A huge number of images was recorded but, due to some technical problems occurred to the optical system during tests, the quality of the images was not very good and the automated method of image analysis reported in [16] was not applicable. Therefore, images have been analysed with the naked eye and the correspondence between colours and thickness has been roughly estimated using the classical calibration table as the one reported in [17] that takes into account that the disc has a semi-reflecting chromium layer and a protective $\mathrm{SiO}_{2}$ coating of about $0.1 \mu \mathrm{m}$. Even if the precision of the measurements is not very high being affected by the human perceptivity, it can be considered sufficient for the purpose of this work.

For increasing the level of confidence in the experimental data, the values of the central film thickness, $\mathrm{h}_{\mathrm{c}}$, have been also calculated using one of the classical formulas for isothermal conditions [18]:

$h_{c}=3.61\left[\left(\eta_{0}^{0.68} \alpha^{0.53} u^{0.68} R_{x}^{0.446}\right)\left(1-0.61 e^{-0.73 k}\right)\right] /\left[E^{10.087} F^{0.063}\right\rfloor$ 
where $\eta_{0}$ is the lubricant viscosity at ambient pressure, $\alpha$ the piezoviscosity coefficient, $u$ the entraining velocity, $R_{x}$ the radius of the spherical specimen, $k$ the eccentricity ratio $(k=1$ in this case $), E^{\prime}=2[(1-$ $\left.\left.\left.v_{\mathrm{s}}^{2}\right) / \mathrm{E}_{\mathrm{s}}+\left(1-v_{\mathrm{d}}^{2}\right) / \mathrm{E}_{\mathrm{d}}\right)\right]^{-1}$ the equivalent elastic modulus $\left(\mathrm{E}_{\mathrm{s}}, \mathrm{E}_{\mathrm{d}}, v_{\mathrm{s}}\right.$ and $v_{\mathrm{d}}$ Young and Poisson moduli of specimen and disc) and $\mathrm{F}$ the load.

Thermal effects can be included using a reduced value for the central film thickness obtained multiplying $\mathrm{h}_{\mathrm{c}}$ by a dimensionless reduction factor $\Phi$. Several formulas for this factor are available in literature. Just to be a little more confident with the results, two of them have been used, the ones reported in [18] and [19], respectively:

$$
\begin{aligned}
& \Phi=\left\lfloor 1-13.2\left(\mathrm{p}_{\mathrm{H}} / \mathrm{E}^{\prime}\right) \mathrm{L}^{0.42}\right\rfloor /\left\lfloor 1+0.213\left(1+2.23 \mathrm{~S}^{0.83}\right) \mathrm{L}^{0.64}\right\rfloor \\
& \Phi=1 /\left\lfloor 1+0.1\left(1+8.33 \mathrm{~S}^{0.83}\right) \mathrm{L}^{0.64}\right\rfloor
\end{aligned}
$$

where $\mathrm{p}_{\mathrm{H}}$ is the Hertzian pressure and $\mathrm{L}=\beta \eta_{0} \mathrm{u}^{2} / \kappa$ the dimensionless thermal loading parameter, with $\beta=-(\mathrm{d} \eta / \mathrm{dT}) / \eta$ thermo-viscosity coefficient $\left[\mathrm{K}^{-1}\right]$, that, using the Roelands expression for the viscositytemperature variation, can be expressed as $\beta=S_{0} \ln \left(\eta_{0} / \eta_{\infty}\right) /\left(135+\mathrm{T}_{0}\right) \quad\left(\eta_{\infty}=6.3110^{-5} \mathrm{~Pa} \mathrm{~s}\right.$ and $\mathrm{T}_{0}$, reference temperature, expressed in $\left.\left[{ }^{\circ} \mathrm{C}\right]\right)$ and $\kappa=\mathrm{A}-\mathrm{B}\left(\mathrm{T}_{0}+273.1\right)$ thermal conductivity $[\mathrm{W} /(\mathrm{m} \mathrm{K})]$. For the lubricant used, $\mathrm{S}_{0}=1.07, \mathrm{~A}=0.1678 \mathrm{~W} /(\mathrm{m} \mathrm{K})$ and $\mathrm{B}=0.0001094 \mathrm{~W} /\left(\mathrm{m} \mathrm{K}^{2}\right)$.

Due to the very low value of $\mathrm{L}$ for the experimental condition tested (its maximum values, that occurs for the results presented in this work for $\mathrm{u}=0.4 \mathrm{~m} / \mathrm{s}$, is about $310^{-3}$ ), which means that thermal effects are not very significant with respect to the film thickness, the two formulas give almost the same results (the biggest difference, that occurs for the biggest specimen at the highest speed, is about $2 \mathrm{~nm}$ ). It is worth noting that the above formulas furnish the same results for positive and negative values of $S$ (the absolute value should be used for negative values) and do not give a unitary value for $S=0$. Therefore these formulas are not able to predict the different values of the film thickness for positive and negative values of $S$ found for instance in [3] and [13].

Some calculated values of the central film thickness under isothermal conditions are compared in Tab.2 with the ones obtained with the greater thermal correction, obtained for $S=1.8$, and the ones estimated by

\begin{tabular}{|c|c|c|c|c|c|c|c|c|c|}
\hline \multirow[t]{2}{*}{ Specimen $=>$} & \multicolumn{3}{|c|}{ S1 } & \multicolumn{3}{|c|}{ S3 } & \multicolumn{3}{|c|}{ S6 } \\
\hline & is & th & ex & is & th & ex & is & th & ex \\
\hline $\mathrm{u}=0.1 \mathrm{~m} / \mathrm{s}$ & 0.051 & 0.050 & 0.04 & 0.075 & 0.074 & 0.07 & 0.112 & 0.111 & 0.12 \\
\hline $\mathrm{u}=0.2 \mathrm{~m} / \mathrm{s}$ & 0.081 & 0.080 & 0.08 & 0.120 & 0.118 & 0.12 & 0.179 & 0.176 & 0.19 \\
\hline $\mathrm{u}=0.4 \mathrm{~m} / \mathrm{s}$ & 0.130 & 0.126 & 0.13 & 0.192 & 0.185 & 0.19 & 0.287 & 0.277 & 0.29 \\
\hline
\end{tabular}
the rough analysis of the interference images for $S=1.8$.

Table 2. Central film thickness, $h_{c}[\mu \mathrm{m}]$, for three values of the entraining velocity $u$ calculated under isothermal conditions (is) and with maximum thermal correction $(\mathrm{S}=1.8)$ (th); estimated values from interference images for $\mathrm{S}=1.8(\mathrm{ex})$.

The differences between the film thickness values without and with thermal correction increase obviously by increasing the speed, but they are always of the order of a few nanometers with a maximum of $10 \mathrm{~nm}$ for the biggest specimen (S6) at the highest speed. The differences among the theoretical and the experimental values are of about the same order of magnitude.

Just to give a hint, some interferograms recorded for the specimen $\mathrm{S} 1$ are shown in Fig.5 (the side of each images is $0.6 \mathrm{~mm}$ ). For the tested values, the main differences occur by varying the velocity, but also some variations in colours can be noted by varying $\mathrm{S}$. These differences are more evident for the smallest specimen because colour differences in interference images are bigger for lower film thickness. For the highest velocities of the specimen some vibrations occur that explain the different dimension of the deformed contact area for $\mathrm{S}=+1.8$. 
The variations connected to the use of the two different shafts are not very significant, as it can be noted by comparing the images obtained for $\mathrm{S}=+1.8$ of Fig. 5 and Fig.6.

The differences, both by varying S and the shaft, are negligible for the specimen S3 and S6. The recorded images by varying both $S$ and the shaft are in these cases always very similar to the sample interferograms that are shown in Fig.7 and Fig.8, reported just for the sake of completeness (the same magnification of the previous figures is used).

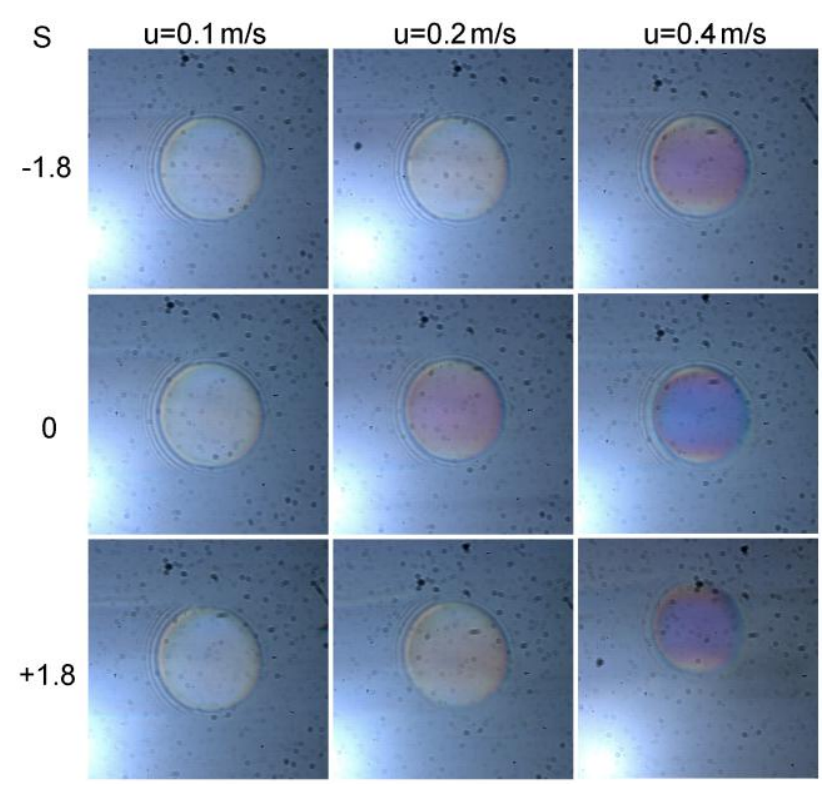

Fig. 5. Interference images recorded for three values of the entraining velocity $u$ and three values of the slide-to-roll ratio S. Specimen S1, steel shaft with the ceramic insert.

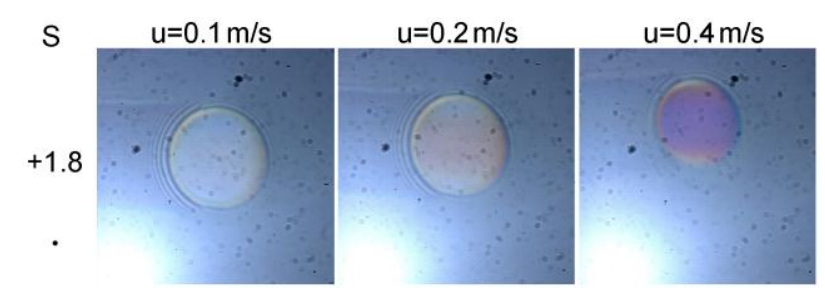

Fig. 6. Interference images recorded for three values of the entraining velocity $u$ and $S=1.8$. Specimen S1, steel shaft.

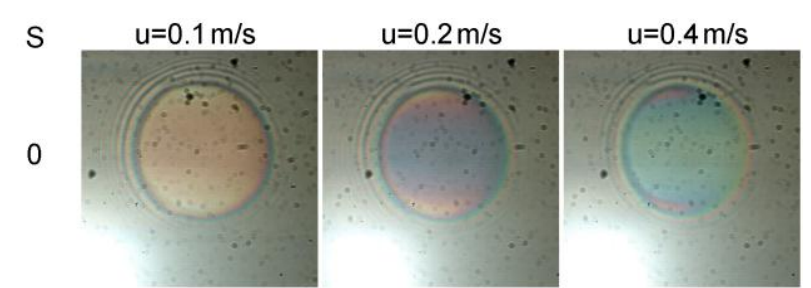

Fig. 7. Interference images recorded for three values of the entraining velocity u and $S=0$. Specimen $S 3$, shaft with the ceramic insert.

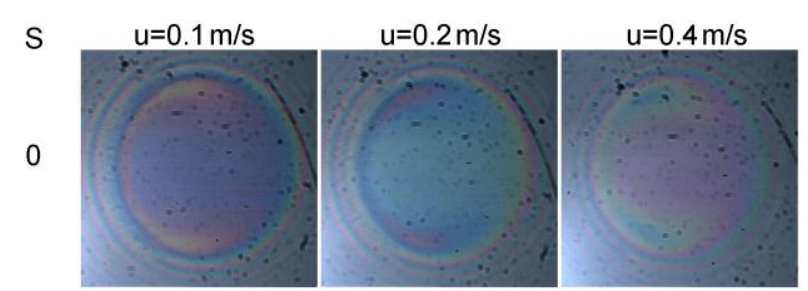

Fig. 8. Interference images recorded for three values of the entraining velocity u and $\mathrm{S}=0$. Specimen $\mathrm{S} 6$, shaft with the ceramic insert. 
Note that, for the working conditions used in this work, the dimple, evident for instance in the results shown in [12], does not seem to be present in an evident manner, most probably because of the much lower viscosity of the lubricant than in the previous works.

\section{Discussion}

The differences between the friction results obtained with the two different shafts can be better evidenced by grouping the results in other manners than the one used in Fig.4, as shown in Fig.9 and Fig.10.

Each diagram in Fig.9 refers to results obtained for a single value of $S$ with all specimens and shafts.
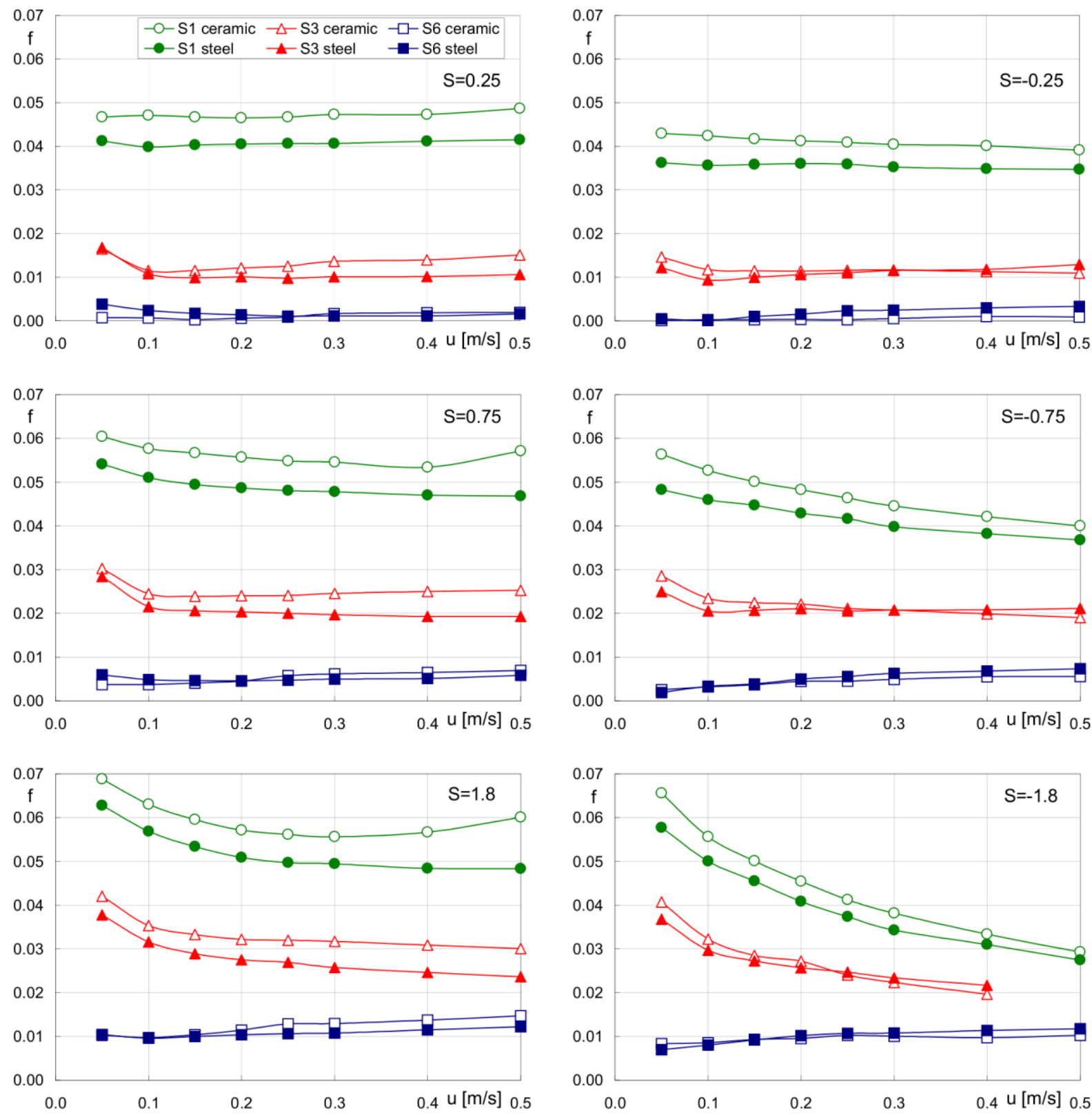

Fig. 9. Measured friction trends as a function of the entraining velocity $\mathrm{u}$ for three positive (diagrams on the left) and negative (diagrams on the right) values of the slide-to-roll ratio $S$ for all specimens and shafts tested.

It is evident that the friction values measured when the shaft with the ceramic insert is used are higher than when the steel shaft is used. It is also evident that this effect is bigger when the rotational speed of the shaft is higher, which corresponds to smaller dimensions of the specimens. As already mentioned above, this can be put in relation to the different temperatures of the specimens proven by some rough measurements made with a thermocouple put in contact with each specimen after having completed the 
runs for every speed conditions, immediately after halting the motors. The measured values, reported in Tab.3, give only a rough indication about the increase of the surface temperature of the specimens above the supply oil temperature during the different conditions. However, they clearly indicate some significant differences.

\begin{tabular}{lccc}
\hline Specimen $=>$ & S1 & S3 & S6 \\
\hline steel, $S>0$ & 12.0 & 6.3 & 2.2 \\
ceramic, $S>0$ & 6.5 & 3.4 & 1.8 \\
steel, $S<0$ & 3.9 & 3.3 & 1.6 \\
ceramic, $S<0$ & 3.3 & 1.7 & 1.6 \\
\hline
\end{tabular}

Table 3. Temperature increase of the specimens, $\Delta \mathrm{T}\left[{ }^{\circ} \mathrm{C}\right]$, measured at the end of each test session with the steel shaft and the one with ceramic insert for positive and negative values of $\mathrm{S}$.

The temperatures measured with the steel shaft are always higher than the ones measured when using the shaft with the ceramic insert. It is evident that the heat produced by the rolling bearings and the seals can reach the specimen in a greater amount when the classical steel shaft is used. It is also evident that the heating increases with the rotational speed of the specimens: smaller specimens need to run at higher rotational speed than bigger specimens for reaching the same peripheral velocity (therefore, the biggest effect occurs for the smallest specimen $S 1$ ). In addition, the heating is obviously bigger for $S>0$, when the specimen is running faster than the disc, than for $S<0$, when the disc is running faster than the specimen. However, these variations do not seem to affect the film thickness in a significant way, as evidenced by the interference images recorded. Or, in any case these differences are not of the same order of magnitude of the ones due to the different slide-to-roll ratios or entraining velocities. In presence of a greater heating, the presence of the ceramic insert is more effective as it can be deduced by comparing the diagrams on the left and on the right columns of Fig.9. In fact, the effect of the heat transfer reduction from the shaft to the specimen and from the specimen to the lubricant inside the contact is greater for positive values of $\mathrm{S}$.

Looking at the slope of the friction curves, it can be noted that the trends of $\mathrm{f}$ with $\mathrm{u}$ tend to become more descending by increasing the absolute value of $S$ and by decreasing the specimen dimensions; this effect is also greater for negative than for positive values of $\mathrm{S}$. All these three aspects evidence that the descending trends are related to thermal effects rather than a possible presence of mixed lubrication. A rough verification of the lubrication regime has been made, based on the value of the so called "lambda ratio", $\Lambda$, the ratio between the lubricant film thickness and the equivalent root mean square roughness of the two bodies in contact. The values of $\Lambda$ for the tested conditions are reported in Tab.4. Being the thermal corrections of the film thickness very small, the values have been calculated using the minimum film thickness formulas reported in [18] and the out-of-contact equivalent roughness (the minimum film thickness instead of the central one has been used according with [20]).

\begin{tabular}{lccc}
\hline Specimen => & S1 & S3 & S6 \\
\hline $\mathrm{u}=0.05 \mathrm{~m} / \mathrm{s}$ & 1.3 & 1.4 & 2.2 \\
$\mathrm{u}=0.10 \mathrm{~m} / \mathrm{s}$ & 2.0 & 2.3 & 3.5 \\
$\mathrm{u}=0.15 \mathrm{~m} / \mathrm{s}$ & 2.7 & 3.0 & 4.7 \\
$\mathrm{u}=0.20 \mathrm{~m} / \mathrm{s}$ & 3.3 & 3.7 & 5.7 \\
$\mathrm{u}=0.25 \mathrm{~m} / \mathrm{s}$ & 3.8 & 4.3 & 6.6 \\
$\mathrm{u}=0.30 \mathrm{~m} / \mathrm{s}$ & 4.3 & 4.9 & 7.5 \\
$\mathrm{u}=0.40 \mathrm{~m} / \mathrm{s}$ & 5.2 & 5.9 & 9.1 \\
$\mathrm{u}=0.50 \mathrm{~m} / \mathrm{s}$ & 6.1 & 6.9 & 10.5 \\
\hline
\end{tabular}

Table 4. Dimensionless film thickness $\Lambda$ for several entrainment velocities u evaluated from calculated isothermal minimum film thickness. 
Taking into account that for non-conformal contacts mixed lubrication conditions are usually considered occurring when $\Lambda<2$, mixed lubrication regime could have arisen just for the smallest values of $\mathrm{u}$ and particularly for the smallest specimen $\mathrm{S} 1$. This is confirmed by the absence of wear tracks on the specimens after tests.

The above mentioned intersections of the friction curves in Fig. 4 for negative values of S correspond to the presence of a maximum in the curves where the friction coefficient is plotted against the slide-to-roll ratio, as stated in [14]. Diagrams of this kind are shown in Fig.10, reported just for the sake of completeness.
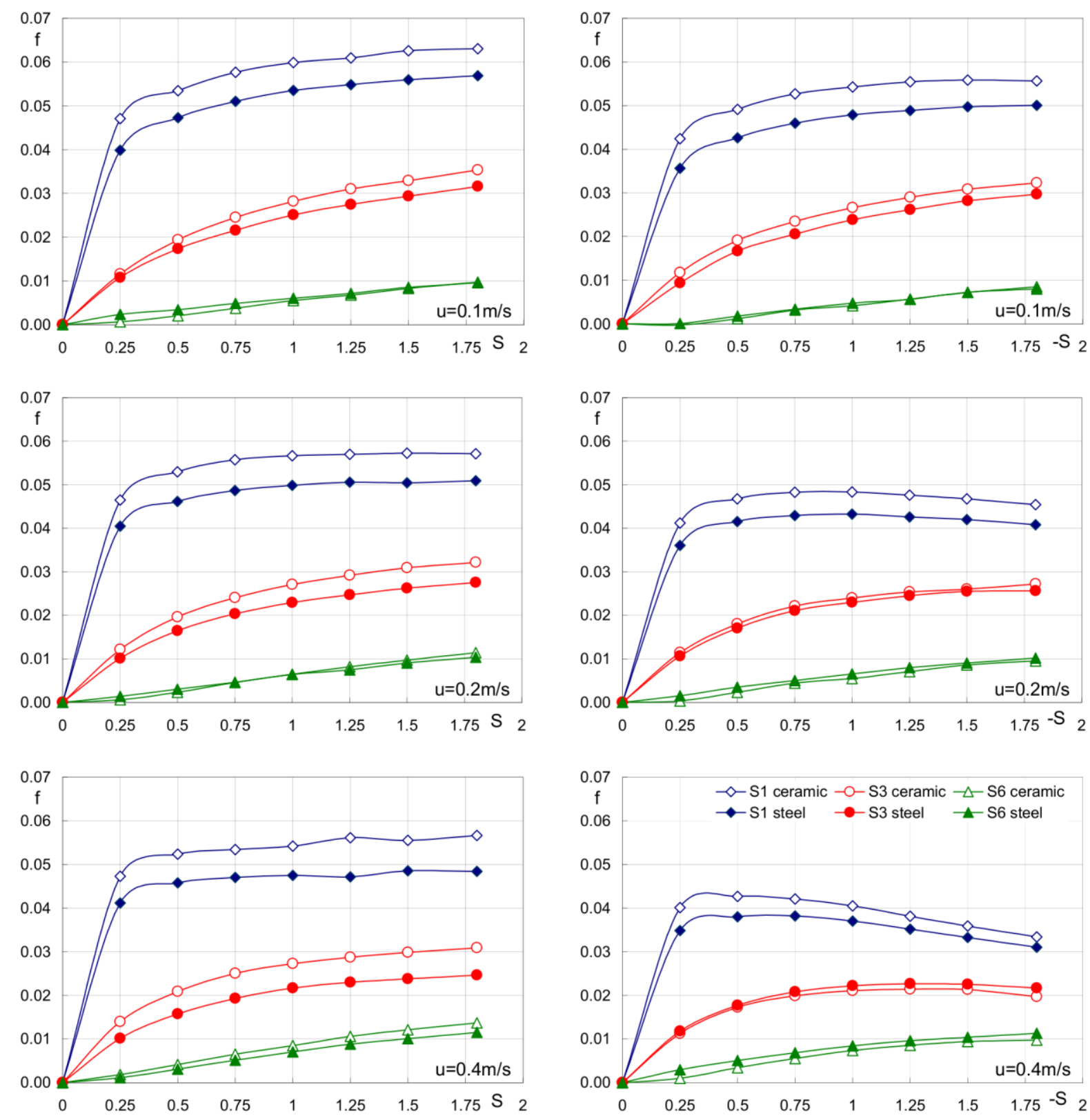

Fig. 10. Measured friction trends as a function of the slide-to-roll ratio $\mathrm{S}$ for three values of the entraining velocity $\mathrm{u}$ obtained for all specimens and shafts tested. Positive values of $\mathrm{S}$ (diagrams on the left) and negative values of $S$ (diagrams on the right).

It is evident that the possibility to have a maximum of the curve increases by decreasing the specimen dimensions and increasing the speed.

Similar considerations to the above ones based on the diagrams of Fig.9 about the influence of the ceramic insert and of the sign of $\mathrm{S}$ could be drawn by analysing the diagrams of Fig. 10 .

All results indicate that the influence of the heating related to the experimental apparatus is not negligible. However, the influence of thermal effects related to the materials of the contacting bodies and of the 
relative movement (the entrainment velocity and the slide-to-roll ratio) appears to be more important at least for the tested values of $\mathrm{u}$ and $\mathrm{S}$.

\section{Conclusions}

Non-conformal lubricated contacts of different geometry have been tested at several rolling speeds and slide-to-roll ratios using spherical specimens of different diameter against a glass disc. In order to evaluate the influence of the experimental apparatus on the results obtained, the same tests have been carried out with two different configurations of the rig: the conventional one, with the specimens connected to a steel shaft, and the modified one with a ceramic element inserted in the shaft specifically designed and realized for limiting the transfer of the heat generated by the shaft seals and supporting bearings to the lubricated contact.

Three spherical specimens of different dimensions have been tested under the same working conditions with both shafts. It has been found that the surface temperature of the contacting bodies influences the friction value in a not negligible manner. The use of the shaft with the ceramic insert produce a reduction in the specimen surface temperature and an overall increase in the friction developed in the lubricated contact that is probably due to a local increment in the lubricant viscosity. Greater influence on friction has been detected for the smallest specimen that experiences a major heating from the bearing and seals due to the higher rotation speed necessary to reach the same peripheral velocity.

The heating of the lubricant inside the contact zone does not seem to affect particularly the film thickness, as evidenced by the analysis of the interference images of the contact recorded during tests. This is consistent with the biggest influence of the inlet lubricant viscosity for the film thickness.

For the experimental conditions tested, the configuration of the experimental apparatus influences the absolute value of friction but the shapes of the friction curves as a function the entraining velocity and of the slide-to-roll ratio are not altered in a significant way. Therefore, some particular findings as the intersections of the friction curves in the Stribeck-like diagrams for negative values of the slide-to-roll ratio (corresponding to glass disc velocities greater than the ones of the steel specimen) and the different values of friction for positive and negative values of the slide-to-roll ratio are confirmed to be more related to thermal effects inside the lubricated contact, as the viscosity wedge action, than to the configuration of the experimental apparatus.

Finally, it could be worthy to remark that the temperature of the contacting bodies is not only influenced by what is occurring inside the EHL contact but also by the surrounding conditions related to the structure and to the working conditions of an experimental apparatus as well as of a real machine. Thus friction values obtained with an experimental apparatus should be always used taking into account that the level of confidence of the results depends on the similarity between the rig and the real machine.

\section{Acknowledgements}

The author would like to thank Dr. Marco Lorenzetti and Ing. Giacomo Bassi for the experimental work carried out.

\section{References}

[1] Wilson WRD, Sheu S. Effects of inlet shear heating due to sliding on elastohydrodynamic film thickness. ASME Journal of Lubrication Technology 1983; 105:187-188.

[2] Olver AV. Testing transmission lubricants: the importance of thermal response. Journal Aero. Eng., Proc.Instn. Mech. Engrs 1991; 205: 35-44.

[3] Smeeth M, Spikes HA The influence of slide/roll ratio on the film thickness of an EHD contact operating within the mixed lubrication regime. Proceedings of the 22th Leeds-Lyon Symposium on Tribology, 1995. Oxford: Elsevier 1996; p. 695-703. 
[4] Kaneta M. For the establishment of a new EHL theory. Proceedings of the 25th Leeds-Lyon Symposium on Tribology, 1999. Oxford: Elsevier 2000; 25-36.

[5] Yang P, Qu S, Kaneta M. Formation of steady dimple in TEHL contacts, ASME J Tribology 2001; 123:42-49.

[6] Guo F, Wong PL. An anomalous EHL film: inlet dimple, ASME J Tribology 2005; 127:425-434.

[7] Kaneta M, Yang P. Effects of thermal conductivity of contacting surfaces on point EHL contacts. ASME J Tribology 2003; 125:731-738.

[8] Reddyhoff T, Spikes HA, Olver AV. Improved infrared temperature mapping of elastohydrodynamic contacts. Proc. IMechE Part J 2009; 223: 1165-1177.

[9] Clarke A, Sharif KJ, Evans HP, Snidle RW. Heat partition in rolling/sliding elastohydrodynamic contacts. ASME J Tribology 2006; 128: 67-78.

[10] Yang P, Liu X. Effects of solid body temperature on the non-Newtonian thermal elastohydrodynamic lubrication behaviour in point contacts. Proc. IMechE Part J 2009; 223: 959969.

[11] Brandão JA, Meheux M, Seabra JHO, Ville F, Castro MJD. Traction curves and rheological parameters of fully formulated gear oils. Proc. IMechE Part J 2011; 225: 577-593.

[12] Bassani R, Ciulli ., Carli M, Stadler K. Experimental investigation of transient and thermal effects on lubricated non-conformal contacts. Tribotest 2007; 13: 183-194.

[13] Ciulli E, Stadler K, Draexl T. The influence of the slide-to-roll ratio on the friction coefficient and film thickness of EHD point contacts under steady state and transient conditions. Tribology International 2009; 42(4): 526-534.

[14] Ciulli E, Lorenzetti M, Stadler K. Investigation on material and dimension effects in elastohydrodynamic lubricated point contacts. Proceedings of the 16th International Colloquium Tribology, Technische Akademie Esslingen, Germany, 2008; 11p., on CD.

[15] Ciulli E, Lorenzetti M, Vela D. Influence of the experimental apparatus on thermal effects in elastohydrodynamic lubricated contacts. Proceedings of ECOTRIB 2009, 2nd European Conference on Tribology, Pisa, Italy, June 7-10, 2009, Edizioni ETS, Pisa, ISBN 978-884672426-7, Vol.2, p. 591-596.

[16] Ciulli E, Draexl T, Stadler K. Film thickness analysis for EHL contacts under steady-state and transient conditions by automatic digital image processing, Advances in Tribology 2008; Article ID 25187, 16 pages, 2008. doi:10.1155/2008/25187, http://www.hindawi.com/journals/at/volume2008/, ISSN: 1687-5915, e-ISSN: 1687-5923, doi:10.1155/AT.

[17] Bassani R, Ciulli E, Stadler K, Carli M. Lubricated non-conformal contacts under steady-state and transient conditions. XVII Congresso AIMETA, Firenze, 11-15 Settembre 2005, Firenze University Press 2005; ISBN 88-8453-248-5, 11p., on CD.

[18] Hamrock BJ. Fundamentals of fluid film lubrication, McGraw-Hill, NewYork, 1994.

[19] Castro J, Seabra J. Scuffing and lubricant film breakdown in FZG gears part I. Analytical and experimental approach. Wear 1998; 215: 104-113.

[20] Bassani R, Ciulli E. Friction from fluid-film to boundary lubricated conditions, 29th Leeds-Lyon Symposium on Tribology, in Tribological research and design for engineering systems, Proceedings of the 29th Leeds-Lyon Symposium on Tribology, Leeds, UK, 3-6 September 2002, Elsevier, Amsterdam 2003; pp.821-834, ISBN 0-444-51243-8. 